\title{
Associations between Salivary Cytokines and Periodontal and Microbiological Parameters in Patients undergoing Fixed Orthodontic Appliance Treatment
}

\section{Yong Chen ( $\nabla$ yongchen@xmu.edu.cn )}

Xiamen University Medical College https://orcid.org/0000-0001-8337-4873

\section{Wong Wing Kit}

Department of Dentistry\&Maxillofacial Surgery,United Christian Hosptial

\section{Jayampath C Seneviratne}

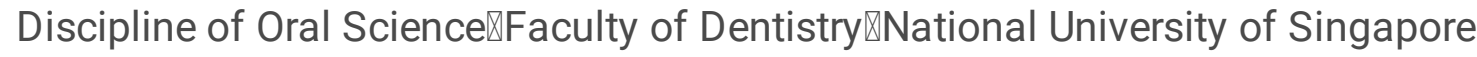

\section{Shuying Huang}

Department of Stomatology $\llbracket$ School of Medicine,Xiamen University

\section{Colman McGrath}

Discipline of Dental Public Health $\varangle$ Faculty of Dentistry $\bigotimes$ The University of Hong Kong

\section{Urban Hagg}

Discipline of Orthodontics $\llbracket$ Faculty of Dentistry $₫$ The University of Hong Kong

\section{Research article}

Keywords: Orthodontics, gingivitis, cytokines, microbiology

Posted Date: November 15th, 2019

DOl: https://doi.org/10.21203/rs.2.17349/v1

License: (c) (i) This work is licensed under a Creative Commons Attribution 4.0 International License.

Read Full License 


\section{Abstract}

Background: Orthodontic treatment can lead to microbial-induced gingival inflammation and aseptic periodontal inflammations. The aim of this study was to investigate the relationship between salivary pro-inflammatory cytokines levels with gingival health status and oral microbe loads among patients undergoing fixed orthodontic appliance treatment (FOAT).

Methods: This was a cross-sectional study among a sample of 111 consecutive orthodontic patients (mean age $18.4 \pm 4.4$ years). Clinical examinations were conducted to assess the gingival health status employing the Modified Gingival Index (MGI), Gingival Bleeding Index (GBI) and Plaque Index (PI). Salivary microbiological assessments of total aerobic and anaerobic bacteria count, streptococci count and lactobacilli count were undertaken. Salivary immunological assessments included Interleukin-1Beta (IL-1 $\beta$ ) and macrophage migration inhibitory factor (MIF) ELISA assays.

Results: The mean \pm standard deviation (SD) of salivary IL- $1 \beta$ was $83.52 \pm 85.62 \mathrm{pg} / \mathrm{ml}$ and MIF was $4.12 \pm 0.96 \mathrm{ng} / \mathrm{ml}$. Positive significant correlations between salivary IL-1 $\beta$ levels and salivary bacteria counts $(r=0.380 \sim 0.446, P<0.001)$, and between salivary MIF levels and total salivary aerobic and anaerobic bacteria counts $(r=0.249 \sim 0.306, P<0.01)$ were observed. A significant positive correlation was found between salivary IL-1 $\beta$ levels and $B I(r=0.216, p<0.05)$.

Conclusions: The levels of salivary IL-1 $\beta$ and MIF correlate with oral bacterial load among patients undergoing fixed orthodontic therapy. This has implications in understanding gingival inflammation/ aseptic periodontal inflammation during orthodontic therapy.

\section{Background}

Previous studies demonstrate that fixed orthodontic appliance treatment (FOAT) creates 'new' locations for plaque retention and increases the risk for gingival inflammation. Thus, the increased potential risk for periodontal diseases is among patients undergoing FOAT is of concern for clinicians[1,2]. Traditionally, assessment or monitoring of gingival conditions has been conducted by clinical examination and expressed by various clinical parameters such as Plaque Index (PI) and Gingival Index (GI)[3].Recently attention has focused on pro-inflammatory cytokines working as biomarkers in periodontal health assessments this has potential in identifying early risk of inflammation and periodontal diseases $[4,5]$. Patients with periodontal diseases have significantly higher levels of Interleukin-1Beta (IL-1 $\beta$ ) in the gingival crevicular fluid (GCF) and in their saliva compared with 'healthy' individuals[6,7].The result of experimental gingivitis study demonstrated a significant positive correlation between Macrophage Migration Inhibitory Factor (MIF) and plaque levels (as assessed by $\mathrm{PI}$ ) and gingivitis (as assessed by $\mathrm{Gl}$ ) [8].Thus, evaluating the levels of pro-inflammatory cytokines may provide insight into risk of periodontal diseases and levels of destructive 'activity' in itself and/or provide a more accurate assessment of periodontal health risks when combined with clinical examinations with implications for clinical practice, prevention and management. 
For those undergoing FOAT, the levels of pro-inflammatory cytokines might be affected not only by the occurrence of microbial induced gingivitis but also by aseptic periodontal inflammations. Previous studies demonstrated that the application of orthodontic forces for teeth moving can induce aseptic inflammatory responses $[9,10] \mathrm{High}$ concentrations of inflammatory cytokines such asIL $-1 \beta$, Interleukin- 6 (IL-6), Interleukin-8 (IL-8) and tumor necrosis factor alpha (TNFa) have been detected in the GCF in both animal models and in human clinical trials[6,11,12].

The assessment of cytokines during FOAT warrants consideration in orthodontic patients in understanding their association and pathways for periodontal health. Furthermore, although the role of the pro-inflammatory cytokines on host inflammatory response to microbial challenges is well recognized, there is a dearth of information on the relationship between pro-inflammatory cytokines and oral microbial load. The aims of the present study were to investigate: 1.the relationship of the salivary pro-inflammatory cytokines levels and gingival health statues, 2 . the relationship of the salivary proinflammatory cytokines levels and oral bacterial loads among patients undergoing FOAT.

\section{Methods}

\section{Participants}

This was a cross sectional study conducting among a consecutive sample of 111 patients (53 male and 58 female, mean age $18.4 \pm 4.4$ years) who were undergoing fixed orthodontic appliance therapy at the Orthodontic Clinic of the Faculty of Dentistry, the University of Hong Kong. Patients were selected based on the following inclusion criteria: (age>13, in good health, non-smoker, no periodontitis (periodontal disease was defined as probing depth $\geq 4 \mathrm{~mm}$ )[13]or other dental diseases that required treatment.

\section{Clinical examinations}

Periodontal health assessments were undertaken employing (i) the Modified Gingival Index (MGI), (ii) Gingival Bleeding Index (GBI) and (iiii) Plaque Index (PI) following a previous protocol[14]: Assessments were carried out on Ramfjord teeth (buccal site of upper right first molar, upper left central incisor, upper left first premolar, lower left first molar, lower right central incisor, lower right first premolar[15].A single trained and calibrated examiner performed all clinical examinations.

\section{Microbiological and immunological examinations}

Saliva samples were obtained: No food or drink was permitted for $2 \mathrm{~h}$ before collection. During the sample collection, the volunteers remained in a seated position, with their head tilted forward. The procedure was accomplished in a quiet and well-ventilated room.Saliva samples (unstimulated) were collected from the participants after clinical examinations. Initially, the examiner instructed each participant to rinse with 
distilled water once, and then collect unstimulated saliva produced during a 5-min period with a sterile plastic bottle[16].

Microbiological assessments: Saliva samples held for culture were packed in ice and were plated within 2 hours in the very day of collection. Serial 10 -fold dilutions were made and $0.05 \mathrm{ml}$ of each dilution was inoculated onto horse blood agar (HBA) plates $\S$ for aerobic culture and anaerobic culture, and MitisSalivarius agar[17]" and Rogosa agar[18] "were used for selective culture streptococci and lactobacilli. Inoculum was spread evenly over the agar with use of a spiral plater. The aerobic cultures and anaerobic cultures were incubated for 48 hour. Colony counts were performed on plates yielding 30 to 300 bacterial colonies per plate[19].The number of viable bacteria per milliliter was calculated by multiplying the number of colonies by the reciprocal of the dilution factor.

Immunological assay: Saliva samples held for IL-1Beta and MIF assay were collected and immediately transferred to Eppendorf tube and frozen at $-70^{\circ} \mathrm{C}$. Before enzyme linked immunosorbent assay (ELISA), collected saliva samples were thawed and centrifuged at $10,000 \mathrm{rpm}$ for $5 \mathrm{~min}$ to precipitate bacteria and other impurities, and then the supernatant was used for assay. This assay employed the quantitative sandwich enzymeimmuno assay technique. The supernatants were diluted with the reagent diluents within the ELISA kit\# in the ratio of 1:2 for IL-1Beta and 1:4 for MIF. Standard curves were generated as manufacture instruction: recombinant human IL-1Beta and MIF were series diluted in reagent diluents and developed for each set of samples assayed (Figure 1,2). The results were reported within the linearity of the assay. The values obtained were multiplied by the dilution factor so as to obtain the actual concentration of salivary cytokines. The results were reported as concentration of cytokines in pictogram or nanogram per milliliter. All ELISA determinations were performed in duplicate.

\section{Statistical analysis}

Statistical analysis was performed by SPSS $17.0,{ }^{* *}$ Pearson correlation and Spearman correlation test was used to assess the strength of association between salivary IL-1Beta and MIF levels and clinical and microbiological parameters, respectively. Multiple linear regressions were used to analyze the effects of the correlated parameters on salivary IL-1Beta and MIF levels. The level of significance was set at $\mathrm{p}<0.05$.

\section{Results}

Table 1 provides information on sample demographic, microbiological and clinical parameters of all participants, the mean and standard deviation (SD) of clinical indices indicated most of the participants show a mild to moderate level of gingivitis. Detectable levels of saliva bacteria were found in all saliva samples in following culture mediums or conditions: Blood agar (Aerobic and Anaerobic) and Mitis Salivarius agar. In 20 saliva samples, no lactobacilli were detected in Rogosa agar culture. Detectable levels of IL $-1 \beta$ and MIF were found in all saliva samples. 
The correlation between participants' salivary IL-1 $\beta$ and MIF levels and the microbiological and clinical parameters shown in Table 2, Positive significant correlations were observed between salivary IL-1 $\beta$ levels and $\mathrm{GBI})(r=0.216, p<0.05)$ and between salivary IL-1 $\beta$ levels and salivary bacteria count in different mediums and conditions $(r=0.380 \sim 0.446, p<0.001)$. A positive significant correlation was observed between salivary MIF levels and salivary total aerobic bacteria count and total anaerobic bacteria count $(r=0.249 \sim 0.306, p<0.01)$. Salivary MIF levels were negatively correlated with age $(r=-$ $0.209, p<0.05)$. There was no significant difference in salivary cytokines levels between male and female patients $(p>0.05)$.

Multiple linear regression model investigated the relationships between the correlated clinical and microbiological factors and levels of salivary IL $-1 \beta$ and MIF. Since the total anaerobic bacteria count was highly correlated with total aerobic bacteria count $(r=0.85, p<0.001)$ which suggests these two factors might cause multicollinearity, only the higher correlated factor: total aerobic bacteria count $(r=$ $0.45 p<0.001$ ) was selected for determining salivary $I L-1 \beta$ level in the regression model. Subsequently, the relationship between total aerobic bacteria count, lactobacilli count with salivary IL $-1 \beta$ level according to the following equation: level of salivary $\mathrm{IL}-1 \beta=-497.45+48.65$ total aerobic bacteria count +33.67 lactobacilli count. The association was positive $(p<0.001)$, the results demonstrated that the IL $-1 \beta$ level was directly proportional to total aerobic bacteria count and lactobacilli count (increases with bacteria count), Table 3 . Considering the total anaerobic bacteria count was highly correlated with total aerobic bacteria count which might cause multicollinearity, the relatively higher correlated factor: total anaerobic bacteria count $(r=0.31, p<0.01)$ was selected into the salivary MIF level regression model. Subsequently, the relationship between total anaerobic bacteria count, with salivary MIF level according to the following equation: level of salivary MIF $=530.48$ total aerobic bacteria count. The association was positive $(p<0.001)$, the results demonstrated that the MIF level was directly proportional to total aerobic bacteria count (increases with bacteria count) Table 4.

\section{Discussion}

Although pro-inflammatory cytokines work as biomarkers in periodontal health assessment in general population has been extensively investigated, the relationships between pro-inflammatory cytokines and gingival health statues among orthodontic patients remain unclear. The present cross-sectional study firstly explored the association between the salivary inflammatory mediators and microbiological and clinical features in orthodontic patients. The result shown that there was only a week positive correlation between the salivary $\mathrm{IL}-1 \beta$ and $\mathrm{BI}$, and no other relationship between salivary MIF and IL-1 $\beta$ levels and clinical gingival indices was found, which means both cytokines might not suitable for gingival health assessment among orthodontic patients. Positive correlations were found between salivary IL $-1 \beta$ and MIF levels and total salivary bacteria count in the present study, which indicated that salivary MIF and IL$1 \beta$ levels were mainly in response to bacterial accumulation. This finding confirms that the immunological cytokines play important roles in host inflammatory response to microbial challenges and contributes new knowledge on this research area. 
In the present study, the levels of salivary IL $-1 \beta$ and MIF were selected to explore relationships between immunological, microbiological and clinical parameters among orthodontic patients. IL-1 $1 \beta$ is a key regulator of the host responses to microbial infection and a major modulator of extracellular matrix catabolism and bone resorption[20,21]. It has been reported that salivary levels of IL-1 $\beta$ was positively correlated with BOP in periodontitis patients[22]. MIF is known to be a pro-inflammatory cytokine involved in macrophage and t-cell activation, IgE synthesis, insulin release, carbohydrate metabolism, cell growth and apoptosis, and tumor angiogenesis[22].An experimental gingivitis study reported that the level of MIF positive correlates with plaque index and gingival index[8].These researches indicate that both cytokines are feasible to be used to assess gingival health statues.

However, none of the clinical parameters: $\mathrm{PI}, \mathrm{MGI}, \mathrm{GBI}$ were significantly correlated with salivary MIF level, and there was only a week positive correlation between the salivary IL-1 $\beta$ and GBI in this study. In the multiple regression model, none of the clinical parameters significantly predicted salivary IL $-1 \beta$ levels. These results might due to the effect of orthodontic treatment which could affect cytokines secretion. Previous studies have shown that IL-1 $1 \beta, I L-6, I L-8$ and TNF alpha increased in the GCF when applying orthodontic force[9-11]. Therefore, the secretion of pro-inflammatory cytokines in orthodontic patients is more complicate than that of general population, and pro-inflammatory cytokines working as biomarkers ingingival health status assessment among orthodontic patients requires further study.

The demographic parameters (age, sex, saliva flow rate) did not show a significant association with the salivary IL-1 $\beta$. A negative correlation was found between salivary MIF levels and age, which suggested that salivary MIF level declines with age, which is consistent with a previous study[8]. However, in the multiple regression model, age was not significantly associated with the salivary MIF level $(p>0.05)$. It is plausible that bacteria number is a stronger factor which conceals the effect of age on salivary MIF level with the present sample size.

In this study, positive correlations were found between salivary IL-1 $\beta$ and MIF levels and total salivary bacteria count, and similar correlation coefficients between the total bacteria count (aerobic and anaerobic), streptococci number and lactobacilli number with IL-1 $\beta$ levels which indicates that no specific bacteria combinations might be associated with gingivitis. These results are in general agreement with a previous study, which investigated a larger sample size and reported the detection of multiple pathogenic species in saliva, rather than the presence of any specific pathogens in saliva, which were associated with periodontitis ${ }^{24}$. Although another study reported that higher GCF levels of IL$1 \beta$ corresponded with higher proportions of orange and red complex species in periodontitis patients[23],however, for the subjects in our study who only have gingivitis, specific pathogenic species may not have yet established.

The present study appears to be the first study try to explore the relationship of the salivary proinflammatory cytokines levels and gingival health statues, as well as the relationship of the salivary proinflammatory cytokines levels and oral bacterial loads among patients undergoing FOAT. Our findings indicate that IL-1 $\beta$ and MIF may be useful and appropriate biomarkers to reflect oral bacterial loads. 
However, since this study was a cross-sectional study with a limited sample size, it is premature to extrapolate these results to the general population. Moreover, although the analysis of salivary biomarkers offers advantages such as collection of whole saliva is easy and noninvasive, there are also some disadvantages to using whole saliva for diagnostic purposes, such as saliva contains GCF, oral bacteria, cells and other sources that make identification of the exact site of disease activity difficult[5].Nonetheless further study of the correlations between cytokines levels and oral microbe loads in general population is warranted.

\section{Conclusions}

The levels of salivary IL $-1 \beta$ and MIF correlate with oral bacterial load among patients undergoing fixed orthodontic therapy. This has implications in understanding gingival inflammation/ aseptic periodontal inflammation during orthodontic therapy.

\section{Abbreviations}

FOAT Fixed orthodontic appliance treatment

MGl: Modified gingival Index

GBI: Gingival bleeding index

PI: Plaque index

$I L-1 \beta$ : Interleukin-1Beta, $I L-6$ : Interleukin-6, $I L-8$ : Interleukin-8

TNFa: Tumor necrosis factor alpha

GCF: Gingival crevicular fluid

MIF: Macrophage Migration Inhibitory Factor

ELISA: Enzyme linked immunosorbent assay

HBA: Horse blood agar

$S D$ : Standard deviation

\section{Declarations}

\section{Ethics approval and consent to participate}

This study was approved by The Institution Review Board of The University of Hong Kong /Hospital Authority Hong Kong West Cluster (Reference Number: UW 10-278). All participants were provided written 
informed consent and a written explanationon the background of the study, its objectives, and their involvement. All participants signed the written informed consent, and in the case subjects who were under 18 years old, both the patient and primary care giver were asked to sign the written informed consent.

\section{Consent for publication}

Not applicable

\section{Availability of data and materials}

The datasets used and/or analysed during the current study are available from the corresponding author on reasonable request.

\section{Competing interests}

The authors declare that they have no competing interests.

\section{Funding}

No funding.

\section{Acknowledgements}

Not applicable

\section{Authors' contributions}

$Y C \square W K W \square J C S \square C M G$ and $U H$ designed the study, completed data analysis and quality assessment. YC and SYH drafted the manuscript and substantively revised it.All authors read and approved the final manuscript.

\section{Authors' information}

${ }^{1}$ Associate ProfessorखDepartment of Stomatology, School of Medicine, Xiamen University. yongchen@xmu.edu.cn

${ }^{2}$ Associate ProfessorखDepartment of Dentistry\& Maxillofacial Surgery, United Christian Hospital. drwongwingkit@gmail.com 
${ }^{3}$ Assistant Professor冈Discipline of Oral Sciences, Faculty of Dentistry, National University of Singapore. chaminda_jayampath@nuhs.edu.sg

${ }^{4}$ Master CandidateXDepartment of Stomatology, School of Medicine, Xiamen University. shuyinghuang@yeah.net

${ }^{5}$ Professor冈Discipline of Dental Public Health, Faculty of Dentistry, The University of Hong Kong. mcgrathc@hku.hk

${ }^{6}$ Professor冈Discipline of Orthodontics, Faculty of Dentistry, The University of Hong Kong. euohagg@hku.hk

\section{Footnotes}

$\S$ Oxoid columbia blood base, Oxoid Limited. UK

\| BD Difco ${ }^{\text {TM }}$ Mitis Salivarius Agar, BD,USA

9BD Difco ${ }^{\text {TM }}$ Rogosa SL Agar, BD,USA

\#DuoSet® ELISA Development Kit, R\&D Systems, Inc. USA

**SPSS version 17.0, SPSS, Chicago, IL.USA

\section{References}

1. Diamanti-Kipioti A, Gusberti FA, Lang NP. Clinical and microbiological effects of fixed orthodontic appliances. J Clin Periodontol. 1987;

2. Pandis N, Papaioannou W, Kontou E, Nakou M, Makou M, Eliades T. Salivary Streptococcus mutans levels in patients with conventional and self-ligating brackets. Eur J Orthod. 2010;

3. Hasegawa K, Furuichi Y, Shimotsu A, Nakamura M, Yoshinaga M, Kamitomo M, et al. Associations Between Systemic Status, Periodontal Status, Serum Cytokine Levels, and Delivery Outcomes in Pregnant Women with a Diagnosis of Threatened Premature Labor. J Periodontol. 2003;

4. Jaedicke KM, Preshaw PM, Taylor JJ. Salivary cytokines as biomarkers of periodontal diseases. Periodontology 2000. 2016.

5. Miller CS, King CP, Langub MC, Kryscio RJ, Thomas M V. Salivary biomarkers of existing periodontal disease: A cross-sectional study. J Am Dent Assoc. 2006;

6. Gamonal J, Acevedo A, Bascones A, Jorge O, Silva A. Levels of Interleukin-1 $\beta,-8$, and -10 and RANTES in Gingival Crevicular Fluid and Cell Populations in Adult Periodontitis Patients and the Effect of Periodontal Treatment. J Periodontol. 2000; 
7. Javed F, Al-Kheraif AA, Al Amri MD, Mikami T, Vohra F, Warnakulasuriya S, et al. Periodontal Parameters and Whole Salivary Cytokine Profiles Among Habitual Gutka Chewers and Non-Chewers. J Periodontol. 2015;

8. Nonnenmacher C, Helms K, Bacher M, Nüsing RM, Susin C, Mutters R, et al. Effect of age on gingival crevicular fluid concentrations of MIF and PGE2. J Dent Res. 2009;

9. Ren Y, Vissink A. Cytokines in crevicular fluid and orthodontic tooth movement. Eur J Oral Sci. 2008;

10. Garlet TP, Coelho U, Silva JS, Garlet GP. Cytokine expression pattern in compression and tension sides of the periodontal ligament during orthodontic tooth movement in humans. Eur J Oral Sci. 2007;

11. Bletsa A, Berggreen E, Brudvik P. Interleukin-1a and tumor necrosis factor-a expression during the early phases of orthodontic tooth movement in rats. Eur J Oral Sci. 2006;

12. Kapoor P, Kharbanda OP, Monga N, Miglani R, Kapila S. Effect of orthodontic forces on cytokine and receptor levels in gingival crevicular fluid: A systematic review. Progress in Orthodontics. 2014.

13. Yoshii S, Tsuboi S, Morita I, Takami Y, Adachi K, Inukai J, et al. Temporal Association of Elevated CReactive Protein and Periodontal Disease in Men. J Periodontol. 2009;

14. Tufekci E, Casagrande ZA, Lindauer SJ, Fowler CE, Williams KT. Effectiveness of an essential oil mouthrinse in improving oral health in orthodontic patients. Angle Orthod. 2008;

15. Mumghamba EGS, Pitiphat W, Matee MIN, Simon E, Merchant AT. The usefulness of using Ramfjord teeth in predicting periodontal status of a Tanzanian adult population. J Clin Periodontol. 2004;

16. Navazesh M, Christensen C, Brightman V. Clinical Criteria for the Diagnosis of Salivary Gland Hypofunction. J Dent Res. 1992;

17. Almståhl A, Wikström M. Oral microflora in subjects with reduced salivary secretion. J Dent Res. 1999;

18. Vallor AC, Antonio MAD, Hawes SE, Hillier SL. Factors Associated with Acquisition of, or Persistent Colonization by, Vaginal Lactobacilli: Role of Hydrogen Peroxide Production. J Infect Dis. 2001;

19. Liewehr FR. Antimicrobial activity of several calcium hydroxide preparations in root canal dentin. $J$ Endod. 2001;

20. Barksby HE, Lea SR, Preshaw PM, Taylor JJ. The expanding family of interleukin-1 cytokines and their role in destructive inflammatory disorders. Clinical and Experimental Immunology. 2007.

21. Engebretson SP, Hey-Hadavi J, Ehrhardt FJ, Hsu D, Celenti RS, Grbic JT, et al. Gingival Crevicular Fluid Levels of Interleukin-1 $\beta$ and Glycemic Control in Patients With Chronic Periodontitis and Type 2 Diabetes. J Periodontol. 2004;

22. Conroy H, Mawhinney L, Donnelly SC. Inflammation and cancer: Macrophage migration inhibitory factor (MIF)-the potential missing link. QJM. 2010.

23. Teles R, Sakellari D, Teles F, Konstantinidis A, Kent R, Socransky S, et al. Relationships Among Gingival Crevicular Fluid Biomarkers, Clinical Parameters of Periodontal Disease, and the Subgingival Microbiota. J Periodontol. 2010; 


\section{Tables}

Table 1 Demographic, microbiological, clinical and immunological parameters of the participants

\begin{tabular}{|c|c|c|c|c|c|}
\hline $\begin{array}{c}\text { Demographic, clinical, microbiological and } \\
\text { immunologicalparameters }\end{array}$ & $\mathbf{N}$ & Mean & SD & Minimum & Maximum \\
\hline Age (years) & 111 & 18.4 & 4.4 & 13.0 & 35.0 \\
\hline Saliva volume (ml/5min) & 111 & 3.1 & 1.6 & 0.7 & 8.9 \\
\hline Plaque Index (PI) & 111 & 1.65 & 0.45 & 0.67 & 2.83 \\
\hline Modified Gingival Index (MGI) & 111 & 1.64 & 0.41 & 0.67 & 2.67 \\
\hline Bleeding Index (BI) & 111 & 0.50 & 0.38 & 0.00 & 1.67 \\
\hline Total aerobic bacteria countaLog10/ml) & 111 & 6.98 & 0.49 & 5.30 & 7.89 \\
\hline Total anaerobic bacteria countaLog10/ml) & 111 & 7.03 & 0.49 & 5.30 & 7.91 \\
\hline Streptococci countlLog10/ml) & 111 & 5.19 & 0.57 & 3.30 & 6.08 \\
\hline lactobacilli countᄆLog10/ml) & 91 & 4.05 & 0.66 & 2.30 & 5.09 \\
\hline 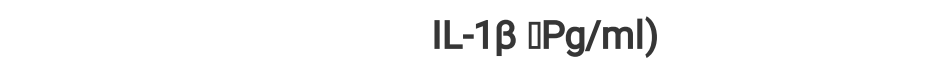 & 111 & 83.52 & 85.62 & 3.99 & 471.16 \\
\hline MIF ng/ml) & 111 & 4.12 & 0.96 & 1.87 & 6.04 \\
\hline
\end{tabular}

Table 2 The Pearson correlation of demographic, microbiological, clinical parameters and salivary IL-1 $\beta$ and MIF levels. 


\begin{tabular}{|c|c|c|c|c|}
\hline Demographic, Clinical and Microbiological Parameters & \multicolumn{2}{|c|}{ IL-1 Beta } & \multicolumn{2}{c|}{ MIF } \\
\cline { 2 - 5 } & Correlation & P & Correlation & P \\
\hline Age & -0.176 & 0.065 & -0.209 & 0.028 \\
\hline Saliva Volume (ml/5min) & 0.089 & 0.352 & -0.108 & 0.257 \\
\hline Plaque Index (PI) & 0.065 & 0.495 & 0.022 & 0.819 \\
\hline Modified Gingival Index (MGI) & 0.167 & 0.080 & 0.082 & 0.390 \\
\hline Bleeding Index (BI) & 0.216 & 0.023 & 0.013 & 0.896 \\
\hline Total aerobic bacteria countLLog10/ml) & 0.446 & 0.000 & 0.249 & 0.008 \\
\hline Total anaerobic bacteria countDLog10/ml) & 0.426 & 0.000 & 0.306 & 0.001 \\
\hline Streptococci count [Log10/ml) & 0.405 & 0.000 & 0.012 & 0.898 \\
\hline lactobacilli count!Log10/ml) & 0.380 & 0.000 & 0.137 & 0.196 \\
\hline
\end{tabular}

Table 3 Multiple Linear Regression of Salivary IL-1ßlevelsassociated with independent variables ( $r=$ $\left.0.510 ; r^{2}=0.260\right)$

\begin{tabular}{|c|c|c|c|c|c|c|}
\hline \multirow[t]{2}{*}{ Independent Variables } & \multicolumn{2}{|c|}{$\begin{array}{l}\text { Unstandardized } \\
\text { Coefficients }\end{array}$} & \multirow[t]{2}{*}{$t$} & \multirow[t]{2}{*}{ Sig. } & \multicolumn{2}{|c|}{$\begin{array}{c}\text { 95\% Confidence Interval } \\
\text { for B }\end{array}$} \\
\hline & B & Std. Error & & & $\begin{array}{l}\text { Lower } \\
\text { Bound }\end{array}$ & $\begin{array}{l}\text { Upper } \\
\text { Bound }\end{array}$ \\
\hline Intercept & -497.45 & 126.56 & -3.93 & 0.000 & -749.04 & -245.85 \\
\hline Bleeding Index (BI) & 20.83 & 21.10 & 0.99 & 0.326 & -21.11 & 62.77 \\
\hline $\begin{array}{l}\text { Total aerobic bacteria count } \\
\qquad(\log 10 / \mathrm{ml})\end{array}$ & 48.65 & 22.37 & 2.18 & 0.032 & 4.19 & 93.12 \\
\hline Streptococci count (Log10/ml) & 17.85 & 19.03 & 0.94 & 0.351 & -19.99 & 55.69 \\
\hline lactobacilli count (Log10/ml) & 33.67 & 12.95 & 2.60 & 0.011 & 7.92 & 59.42 \\
\hline
\end{tabular}

Table 4 Multiple Linear Regression of Salivary MIF levelsassociated with independent variables $(r=0.340$; $\left.r^{2}=0.116\right)$ 


\begin{tabular}{|c|c|c|c|c|c|c|}
\hline \multirow[t]{2}{*}{ Independent Variables } & \multicolumn{2}{|c|}{$\begin{array}{l}\text { Unstandardized } \\
\text { Coefficients }\end{array}$} & \multirow[t]{2}{*}{$t$} & \multirow[t]{2}{*}{ Sig. } & \multicolumn{2}{|c|}{$\begin{array}{l}\text { 95\% Confidence } \\
\text { Interval for B }\end{array}$} \\
\hline & B & Std. Error & & & $\begin{array}{l}\text { Lower } \\
\text { Bound }\end{array}$ & $\begin{array}{l}\text { Upper } \\
\text { Bound }\end{array}$ \\
\hline Intercept & 999.31 & 1384.01 & .72 & 0.472 & -1744.03 & 3742.65 \\
\hline Age & -32.91 & 20.07 & -1.64 & 0.104 & -72.69 & 6.87 \\
\hline $\begin{array}{l}\text { Total anaerobic bacteria count } \\
\qquad(\log 10 / \mathrm{ml})\end{array}$ & 530.48 & 178.74 & 2.97 & 0.004 & 176.20 & 884.77 \\
\hline
\end{tabular}

Figures

\section{IL-1Beta standard curve}

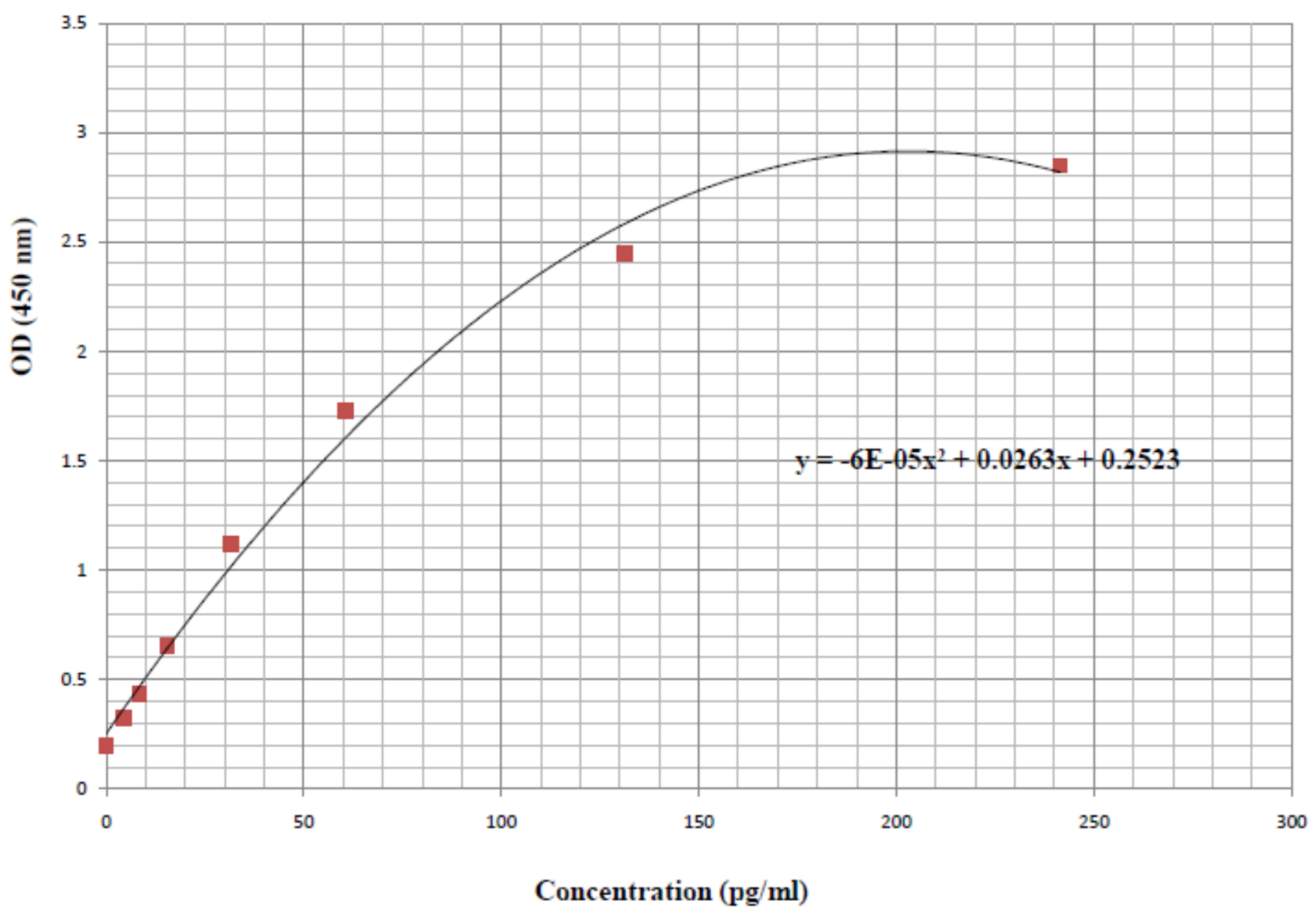


Figure 1

IL-1Beta standard curve

\section{MIF standard curve}

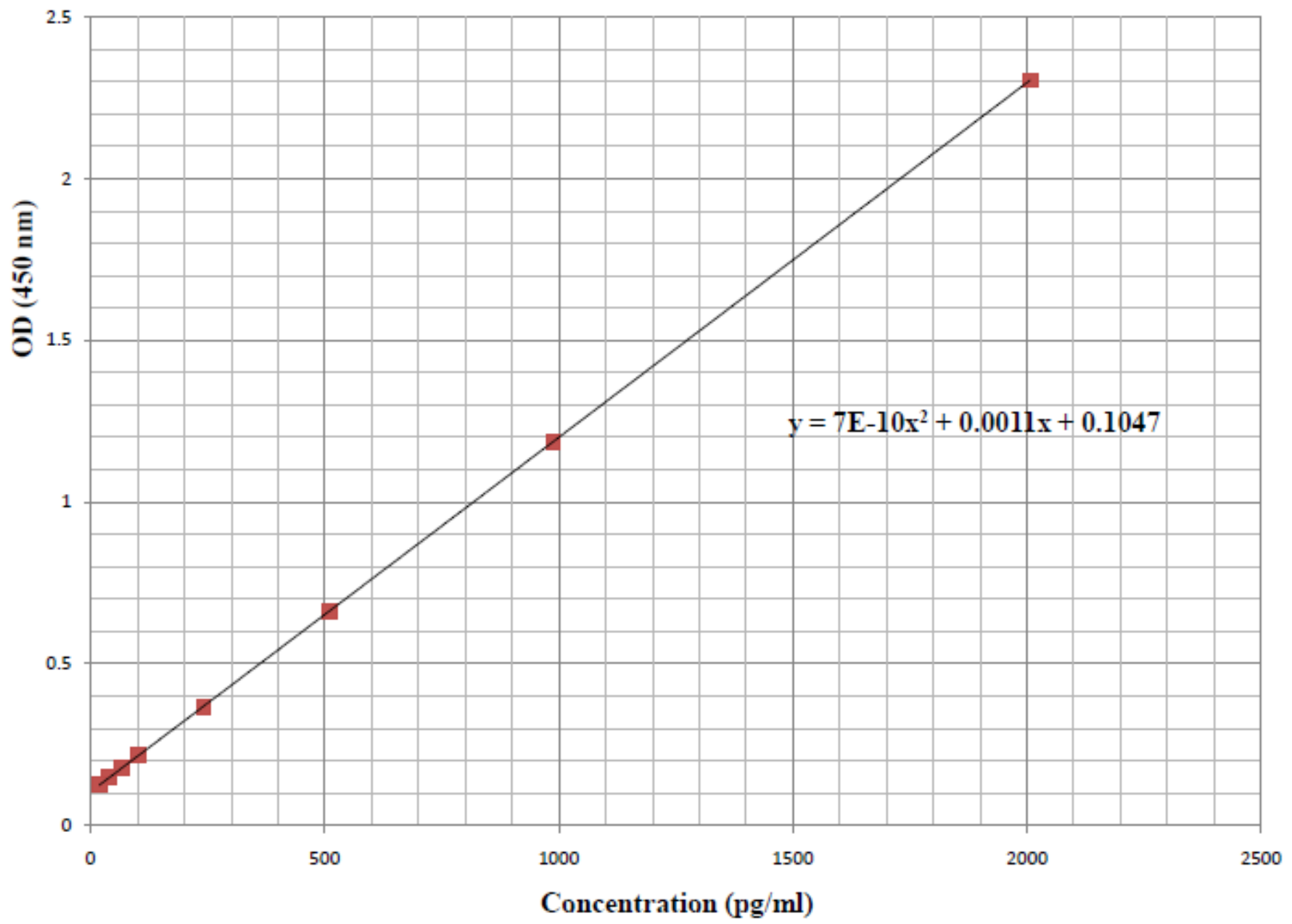

Figure 2

MIF standard curve 